\title{
Image color contrast enhancement using multiscale morphology
}

\author{
Julio César Mello Román ${ }^{a}$, Horacio Legal Ayala ${ }^{a}$ and José Luis Vázquez Noguera ${ }^{a 1}$ \\ ${ }^{a}$ National University of Asuncion, San Lorenzo, Paraguay \\ Received on September 30, 2016 / accepted on October 10, 2016
}

\begin{abstract}
Contrast enhancement aims to improve color images in terms of human interpretation and further processing in other stages of digital image processing. The multiscale mathematical morphology technique is widely used to enhance contrast in grayscale images. The extention for mathematical morphology for color images is not an easy task, because the images can be defined by different color spaces, which are represented by n-dimensional vectors and there is no single way of ordering vectors. HSI color space is used to represent the color of pixels and a lexicographical order $(\mathrm{I} \rightarrow \mathrm{S} \rightarrow \mathrm{H})$ is imposed for processing. The fundamental morphological operations will be extended from this ordering to be applied into color images. In this work, a contrast enhancement algorithm is presented using the features extracted from the color image by the transformed multiscale top-hat. To evaluate the proposed method, various tests were performed using different color images. The experimental results show the effectiveness of the method, in which contrast enhancement was evaluated by the color enhancement factor (CEF) Metric. Also the visual inspection was used.
\end{abstract}

Keywords: Color image, contrast enhancement, multiscale mathematical morphology, color enhancement factor.

\section{Introduction}

Contrast enhancement is a technique used in digital image processing to improve the appearance of images and make them more suitable for human vision. This technique comprises a set of operations that improves the visual quality of the image. These operations allows to enhance the brightness characteristics and the contrast of an image and also intensifies the details present on it.

Contrast enhancement is important because it is useful for further applications such as medical imaging [3], fingerprint recognition [5], geoscience [12], biomedical engineering $[13,11]$ and computer vision [8].

\footnotetext{
${ }^{1}$ E-mail Corresponding Author: jlvazquez@pol.una.py
} 
In digital image processing there are several techniques for contrast enhancement, such as histogram equalization, which improves the contrast of an image by a redistribution of the gray levels [4]; and the multiscale morphology that has shown efficiency in contrast enhancement for grayscale images [2].

In this paper we propose the usage of multiscale mathematical morphology for color images. The main inconvenience is that color images can be defined in different color spaces where pixels are represented by $\mathrm{n}$-dimensional vectors. The HSI color space is adopted because it has compatible characteristics with the perception of human vision [14]. The lexicographical order $\mathrm{I} \rightarrow \mathrm{S} \rightarrow \mathrm{H}$ [7] is used to order the pixels of color images. Furthermore, a contrast enhancement algorithm that applies the top-hat multiscale transform is presented for color images.

The paper is organized as follows: In section 2, the basics of mathematical morphology are presented. In section 3 the proposed algorithm is detailed. In section 4, experimental results are shown using color images from a public database, and section 5 contains the conclusions and future work.

\section{Mathematical morphology}

Mathematical morphology is used by many applications. Its extension to color images is a challenging task, because there is an inconvenience due to the absence of a natural order between vectors that are used to represent pixels of an image [8]. Then the basics of morphological operators are presented below.

Mathematical morphology is based on two basic operations: erosion (minimization) and dilation (maximization).

Given an image $f$ and one structuring element $g$, in which pixels are respectively represented by cartesian coordinates $(u, v)$ and $(s, t)$, where the dilation $(f \oplus$ $g)$ and erosion $(f \ominus g)$ of an image can be defined as:

$$
\begin{aligned}
& (f \oplus g)(u, v)=\max \{f(u-s, v-t)+g(s, t) \mid(u-s, v-t) \in f,(s, t) \in g\} \\
& (f \ominus g)(u, v)=\min \{f(u+s, v+t)-g(s, t) \mid(u+s, v+t) \in f,(s, t) \in g\}
\end{aligned}
$$

For all pixels, the erosion and dilation operations are denoted as $(f \oplus g)$ and $(f \ominus g)$. In order to get the maximum and the minimum using the dilation and erosion operations, color image pixels need to be ordered. Considering that color is represented as vectors, and no natural order is defined for them, the extent of mathematical morphology in color spaces is not trivial, since we need to adopt a color space and an order within this space. In this paper, the HSI color space and the lexicographic order $\mathrm{I} \rightarrow \mathrm{S} \rightarrow \mathrm{H}[7]$ are used to order the pixels. 
The operations of erosion and dilation of de color image with square structuring element $3 \times 3$ using the lexicographical order $\mathrm{I} \rightarrow \mathrm{S} \rightarrow \mathrm{H}$ are illustrated in the Figure 1.

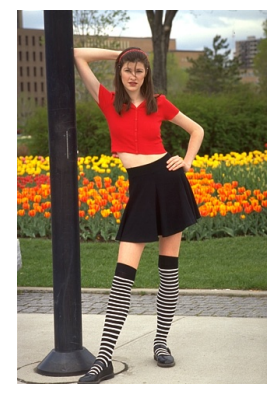

(a)

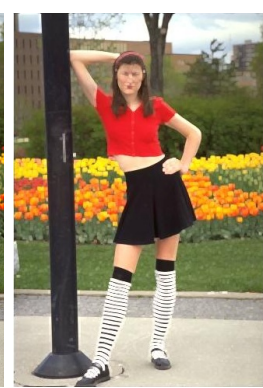

(b)

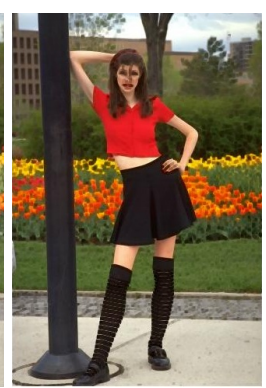

(c)

Figure 1: Original image (a), dilation (b) and erosion (c).

All the mathematical morphology can be extended by the dilation and erosion operations.

The opening $\circ$ and closing $\bullet$ of $f$ by $g$ operations are defined from the concepts of dilation and erosion as follows:

$$
\begin{aligned}
& f \circ g=(f \ominus g) \oplus g \\
& f \bullet g=(f \oplus g) \ominus g
\end{aligned}
$$

The operations of opening and closing of de color image with square structuring element $3 \times 3$ using the lexicographical order $\mathrm{I} \rightarrow \mathrm{S} \rightarrow \mathrm{H}$ are illustrated in the Figure 2.

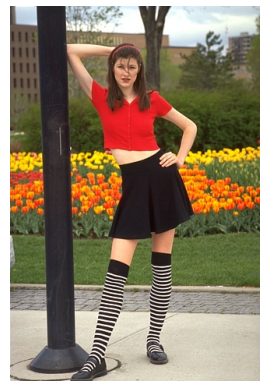

(a)

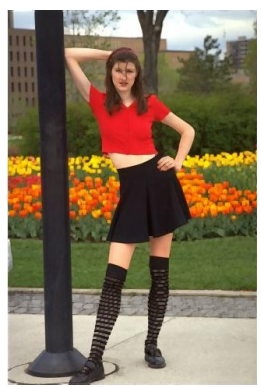

(b)

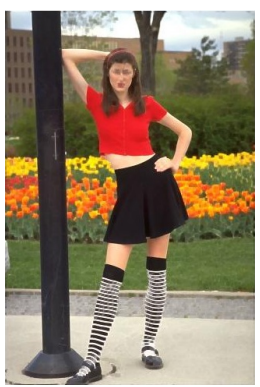

(c)

Figure 2: Original image (a), opening (b) and closing (c). 
Given the opening and closing operations, the white top-hat transform (WTH) and the black top-hat transform $(B T H)$ for an image $f$ are defined as follows:

$$
\begin{gathered}
W T H=f-f \circ g \\
B T H=f \bullet g-f
\end{gathered}
$$

The operations of white top-hat and black top-hat of de color image with square structuring element $3 \times 3$ using the lexicographical order $\mathrm{I} \rightarrow \mathrm{S} \rightarrow \mathrm{H}$ are illustrated in the Figure 3.

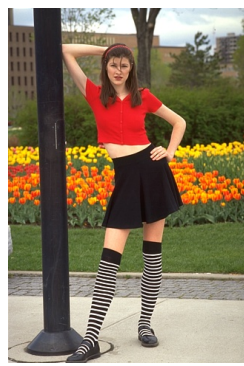

(a)

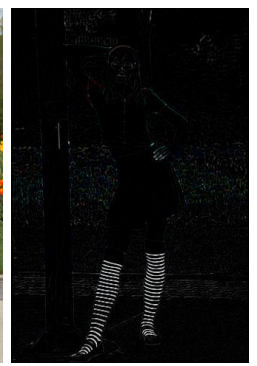

(b)

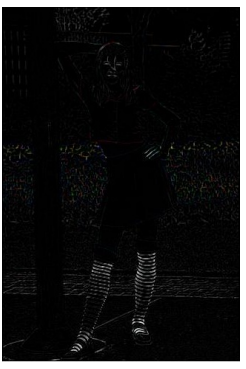

(c)

Figure 3: Original image (a), white top-hat (b) and black top-hat (c).

When using opening and closing operators, the bright and dark areas of the image are attenuated, respectively (WTH obtained bright areas, meanwhile with $B T H$ obtained dark areas of the image).

\section{Proposal}

The contrast enhancement for the image $f$ based on the top-hat transform consists on adding the bright areas of the image $f$ and subtracting the dark areas of the image $f$ as follows [9]:

$$
f_{E}=f+W T H-B T H
$$

where $f_{E}$ is the resulting image with enhanced contrast.

The algorithm proposed in this paper is a variation of the technique described by Bai, Zhou and Xue [2] for grayscale images. They propose a method of contrast enhancement that extracts image features by multi-scale top-hat transform which we call BZX algorithm. Next, to get a better understanding, the method description is exposed. The structuring element is dilated $n-1$ times, where $n$ is the number of iterations: 


$$
\begin{gathered}
g_{1}=g_{1} \\
g_{2}=g_{1} \oplus g_{1} \\
g_{3}=g_{2} \oplus g_{1}=g_{1} \oplus g_{1} \oplus g_{1} \\
\vdots \\
g_{n}=g_{n-1} \oplus g_{1}=\underbrace{g_{1} \oplus g_{1} \oplus g_{1} \oplus \cdots \oplus g_{1}}_{\text {dilated } n-1 \text { times }}
\end{gathered}
$$

First, the bright and dark zones in different scales are obtained, as follows:

$$
\begin{gathered}
W T H_{i}=\left\{\begin{array}{c}
W T H_{1}=f-f \circ g_{1} \\
W T H_{2}=f-f \circ g_{2} \\
W T H_{3}=f-f \circ g_{3} \\
\vdots \\
W T H_{n}=f-f \circ g_{n}
\end{array}\right. \\
B T H_{i}=\left\{\begin{array}{c}
B T H_{1}=f \bullet g_{1}-f \\
B T H_{2}=f \bullet g_{2}-f \\
B T H_{3}=f \bullet g_{3}-f \\
\vdots \\
B T H_{n}=f \bullet g_{n}-f
\end{array}\right.
\end{gathered}
$$

In a second step, the dark and bright zones in different scales differences are obtained as follows:

$$
\begin{gathered}
W T H V_{i}=\left\{\begin{array}{c}
W T H V_{1}=W T H_{2}-W T H_{1} \\
W T H V_{2}=W T H_{3}-W T H_{2} \\
W T H V_{3}=W T H_{4}-W T H_{3} \\
\vdots \\
W T H V_{n-1}=W T H_{n}-W T H_{n-1}
\end{array}\right. \\
B T H V_{i}=\left\{\begin{array}{c}
B T H V_{1}=B T H_{2}-B T H_{1} \\
B T H V_{2}=B T H_{3}-B T H_{2} \\
B T H V_{3}=B T H_{4}-B T H_{3} \\
\vdots \\
B T H V_{n-1}=B T H_{n}-B T H_{n-1}
\end{array}\right.
\end{gathered}
$$

In a third stage, the maximum values for every scale are calculated:

$$
\begin{gathered}
W T H_{\max }=\max _{1 \leq i \leq n} W T H_{i} \\
W T H V_{\max }=\max _{1 \leq i \leq n-1} W T H V_{i} \\
B T H_{\max }=\max _{1 \leq i \leq n} B T H_{i} \\
B T H V_{\max }=\max _{1 \leq i \leq n-1} B T H V_{i}
\end{gathered}
$$


Finally, the contrast enhancement for the image is obtained as follows:

$$
f_{E}=f+\left(W T H_{\max }+W T H V_{\max }\right)-\left(B T H_{\max }+B T H V_{\max }\right)
$$

The variations of the proposed algorithm by Bai, Zhou and Xue involve replacing equations 10 by 13 and 11 by 14 . To get the differences of the bright and dark zones in different scales, the following equations are proposed:

$$
\begin{gathered}
W T H V_{i}=\left\{\begin{array}{c}
W T H V_{1}=W T H_{2}-W T H_{1} \\
W T H V_{2}=W T H_{3}-W T H V_{1} \\
W T H V_{3}=W T H_{4}-W T H V_{2} \\
\vdots \\
W T H V_{n-1}=W T H_{n}-W T H V_{n-2}
\end{array}\right. \\
B T H V_{i}=\left\{\begin{array}{c}
B T H V_{1}=B T H_{2}-B T H_{1} \\
B T H V_{2}=B T H_{3}-B T H V_{1} \\
B T H V_{3}=B T H_{4}-B T H V_{2} \\
\vdots \\
B T H V_{n-1}=B T H_{n}-B T H V_{n-2}
\end{array}\right.
\end{gathered}
$$

Figure 4 shows the proposed algorithm in this article. This gives a better result based on the metric as defined below for images in grayscale and color.

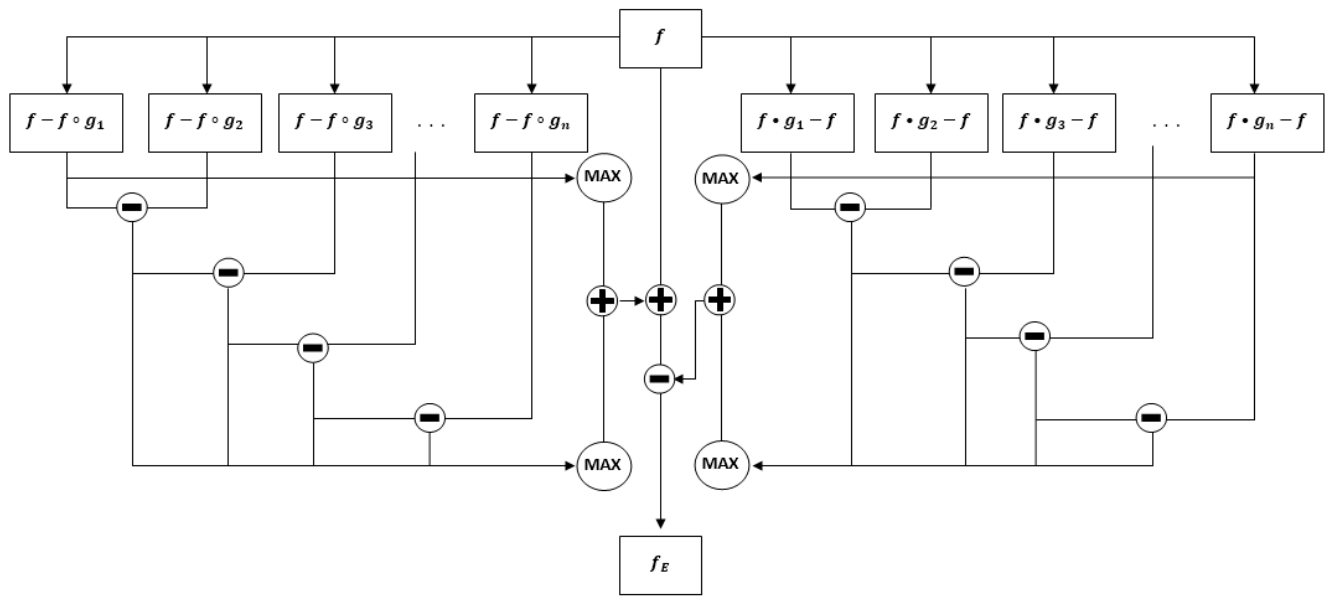

Figure 4: Scheme of the proposed algorithm. 


\section{Results}

In this section the obtained results by the scale algorithms for grayscale and color images will be exposed.

\subsection{Contrast enhancement metrics}

The contrast enhancement metrics are shown to measure the quality of the resulting images.

- Contrast: It is a metric applied to assess the contrast enhancement of grayscale images. Is defined as:

$$
C=\sqrt{\sum_{j=0}^{L-1}(j-E(f))^{2} \times p(j)}
$$

where $j$ represents the value of the pixel $(u, v)$ of the image $f, L$ are the gray levels, $E(f)$ represents the average image intensity and $p(j)$ is the probability of occurrence for the $j$ value. The resulting contrast value, has to be bigger than the original image. This will suppose an improvement.

- Color Enhancement Factor (CEF): This metric is used to evaluate contrast enhancement in color images. This metric quantifies the level of contrast enhancement of an image as mentioned in [10], applied to the image $f$. It is based on the mean and standard deviation of two axes of a simple representation of opposite colors with $\gamma=f_{1}-f_{2}$ and $\beta=\frac{1}{2}\left(f_{1}+f_{2}\right)-f_{3}$, where $f_{1}$ represents the channel $R, f_{2}$ represents the channel $G$ and $f_{3}$ represents the channel $B$ in RGB color space. The equation 16 represents the level of contrast enhancement of the image $f$ as follows:

$$
C M(f)=\sqrt{\sigma_{\gamma}^{2}+\sigma_{\beta}^{2}}+\sqrt{\mu_{\gamma}^{2}+\mu_{\beta}^{2}}
$$

where $\sigma_{\gamma}$ and $\sigma_{\beta}$ respectively correspond to the standard deviation of $\gamma$ and $\beta$. In the same way, $\mu_{\gamma}$ and $\mu_{\beta}$ corresponds to the mean.

Then the $C E F$ is calculated as the rate between the values of $C M\left(f_{E}\right)$ and $C M(f)$ :

$$
C E F=\frac{C M\left(f_{E}\right)}{C M(f)}
$$

where $C M\left(f_{E}\right)$ is the value obtained from the contrast image $f_{E}$ product of applying the equation 16 and $C M(f)$ represents the result of applying the equation 16 , to the original image $f$. If the result is bigger than 1 ; then the 
metric of the equation 17 indicates an improvement in the contrast otherwise there is no improvement.

\subsection{Experimental results}

We used 20 images of a public database [1] for testing both images in grayscale and color images. The input parameters were the original image $f$, the number of iterations $n=7$ and the initial square structuring element $g$ of $3 \times 3$.

The Contrast metric was used to evaluate the results. It is noticeable that both algorithms improves the contrast of the original images in grayscale, but our proposal is better numerically. Figure 5 (a) shows the original image; Figure 5 (b) shows enhanced image with the BZX algorithm and Figure 5 (c) is the enhanced image with the proposed algorithm. The resulting image obtained using the proposed algorithm displays higher brightness and sharpness of details with respect to the original image and the BZX algorithm.

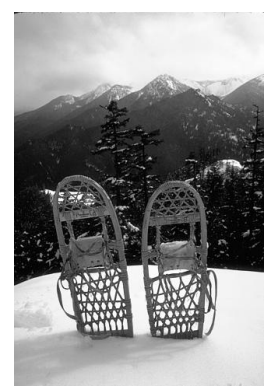

(a) $\mathrm{C}=88.2766$

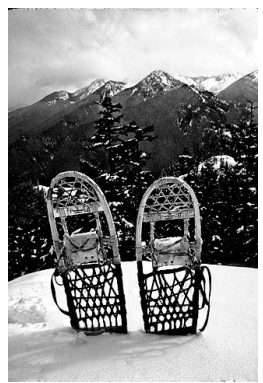

(b) $\mathrm{C}=98.6771$

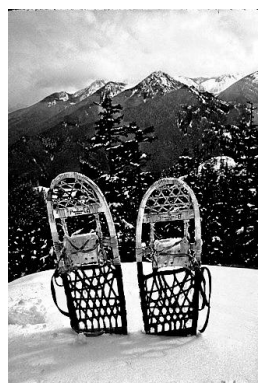

(c) $\mathrm{C}=99.7225$

Figure 5: Visual results obtained by the algorithms for grayscale images.

Also 20 color images were processed, where the $C E F$ metric was used to evaluate the results. The BZX algorithm achieved successful results by a rate of around $80 \%$, while the proposed algorithm had successful results by a rate of $95 \%$. The Figure 6 (a) shows the original image, Figure 6 (b) shows the image obtained with the BZX algorithm and Figure 6 (c) is the image obtained with the proposed algorithm. In the resulting image, higher brightness is achieved compared to the original image and the BZX algorithm.

\section{Conclusions and Future Work}

In this paper, one algorithm for contrast enhancement was presented using the multiscale top-hat transform, which is a variation of the proposal made by Bai, 


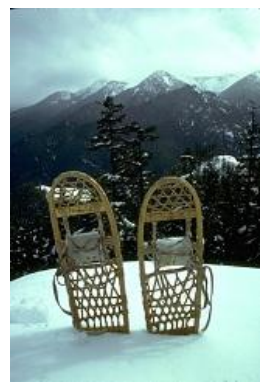

(a)

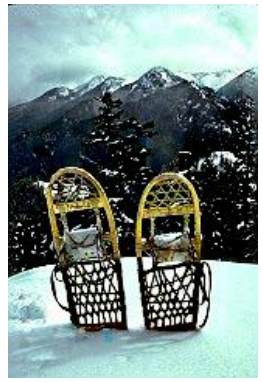

(b) $\mathrm{CEF}=1.0703$

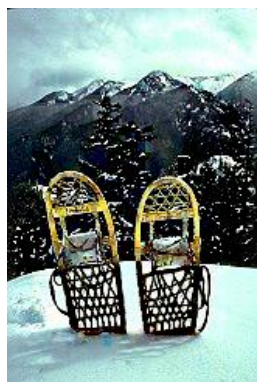

(c) $\mathrm{CEF}=1.1210$

Figure 6: Visual results obtained by the algorithms for color images.

Zhou and Xue [2]. Both algorithms were implemented for grayscale images, as a first step, and then for color images.

The extent of mathematical morphology to color images was performed with the choice of a method of lexicographic order $(\mathrm{I} \rightarrow \mathrm{S} \rightarrow \mathrm{H})$ in the color space HSI.

Experimental results show improvements in contrast for grayscale images according to the metric Contrast, and also in color images according to the metric $C E F$. It is also noteworthy that visual evaluation of resulting images, for the techique proposed here are satisfactory. Therefore the algorithm presented represents a feasible solution for contrast enhancement in color images.

As future work, comparisons can be made in other color spaces, in order analyse differences with other contrast enhancement algorithms.

Acknowledgments. Authors José Luis Vázquez and Horacio Legal wish to acknowledge partial financial support from CONACYT under project \#14-INV202.

\section{References}

[1] Arbelaez, P., Fowlkes, C., \& Martin, D. (2007). The berkeley segmentation dataset and benchmark. see http://www. eecs. berkeley. edu/Research/Projects/CS/vision/bsds.

[2] Bai, X., Zhou, F., \& Xue, B. (2012). Image enhancement using multi scale image features extracted by top-hat transform. Optics \& Laser Technology, 44(2), 328-336. 
[3] Boccignone, G., \& Picariello, A. (1997, April). Multiscale contrast enhancement of medical images. In Acoustics, Speech, and Signal Processing, 1997. ICASSP-97., 1997 IEEE International Conference on (Vol. 4, pp. 27892792). IEEE.

[4] Garg, R., Mittal, B., \& Garg, S. (2011). Histogram equalization techniques for image enhancement. International Journal of Electronics \& Communication Technology, 2(1), 107-111.

[5] Hong, L., Wan, Y., \& Jain, A. (1998). Fingerprint image enhancement: algorithm and performance evaluation. IEEE transactions on pattern analysis and machine intelligence, 20(8), 777-789.

[6] Lambert, P., \& Chanussot, J. (2000). Extending mathematical morphology to color image processing. Proc. CGIP 2000.

[7] Ortiz, F., Torres, F., \& Gil, P. (2004, August). Gaussian noise elimination in colour images by vector-connected filters. In Pattern Recognition, 2004. ICPR 2004. Proceedings of the 17th International Conference on (Vol. 4, pp. 807-810). IEEE.

[8] Ortiz Zamora, F. G. (2002). Procesamiento morfolgico de imgenes en color: aplicacin a la reconstruccin geodsica.

[9] Soille, P. (2013). Morphological image analysis: principles and applications. Springer Science \& Business Media.

[10] Susstrunk, S. E., \& Winkler, S. (2003, December). Color image quality on the internet. In Electronic Imaging 2004 (pp. 118-131). International Society for Optics and Photonics.

[11] Truc, P. T., Khan, M. A., Lee, Y. K., Lee, S., \& Kim, T. S. (2009). Vessel enhancement filter using directional filter bank. Computer Vision and Image Understanding, 113(1), 101-112.

[12] Yang, C., Lu, L., Lin, H., Guan, R., Shi, X., \& Liang, Y. (2008). A fuzzystatistics-based principal component analysis (FS-PCA) method for multispectral image enhancement and display. IEEE Transactions on Geoscience and Remote Sensing, 46(11), 3937-3947.

[13] Yang, G. Z., \& Hansell, D. M. (1997). CT image enhancement with wavelet analysis for the detection of small airways disease. IEEE Transactions on medical imaging, 16(6), 953-961. 
[14] Zamora, F. G. O., Torres-Medina, F., Lopez-Angulo, J., \& Mendez, S. P. (2001, October). Comparative study of vectorial morphological operations in different color spaces. In Intelligent Systems and Advanced Manufacturing (pp. 259-268). International Society for Optics and Photonics. 